\title{
The Effect of Ultrasound on Strain-hardened Metals
}

\author{
Endre Ruszinkó, Ali H. Alhilfi \\ Óbuda University, Népszínház u. 8, H-1081 Budapest, Hungary \\ ruszinko.endre@uni-obuda.hu; alihameed@stud.uni-obuda.hu
}

\begin{abstract}
This paper addresses the case when acoustic energy is applied to plastically deformed metals when the "Ultrasonic recovery phenomenon" is observed, which manifests itself in a decrease of dislocation density, hardness and yield strength. Based on the analysis of the processes occurring in the sonicated material's microstructure, a model has been developed within the terms of the synthetic theory of irrecoverable deformation. We introduce into the equation governing recovery processes a term for expressing the oscillating stress amplitude. The model results show good agreement with the experimental data.
\end{abstract}

\section{Introduction}

Ultrasound energy is an effective tool in metallurgy, metal forming, and many other industry branches and technological processes. With the effects of ultrasound upon metals' deformational properties, the following phenomena can be listed:

(i) Ultrasonic hardening. Acoustic energy propagating through an annealed specimen increases the number of crystalline grid defects - mainly dislocations and vacancies. As a result, the material's yield strength grows as a function of the sonication duration (Fig. 1a). One can see that the yield strength $\sim$ sonication time dependency first increases and then reaches a plateau. The yield strength increment saturation is explained by the gradual restrain of Frank-Read sources caused by dislocations nucleated in preceding cycles. The annihilation of oppositely oriented dislocations emitted by sources on parallel atomic planes also contributes to the gradual decay in the yield strength's increase. Numerous experiments show that the ultrasonic hardening effect is a function of the ultrasonic field's intensity [1]-[4]. 
(ii) Effect of acoustic energy upon the creep characteristics. Creep experiments for materials subjected to the preliminary sonication treatment - sonication + annealing - show that the ultrasonic treatment exerts a positive impact upon the steady-state creep rate. Fig. 1b schematically demonstrates the decrease in the creep rate for copper (one minimum) and aluminum (two minimums) caused by the dislocation substructure formed during the preliminary ultrasonic treatment. As one can see, there are optimal ranges of the sonication duration when the creep rate shows low values, which is due to the thermal stability of the ultrasonic defect structure. Beyond the optimal ranges, the dislocation substructure loses its capacity to impede the creep deformation, and the creep rate tends to its initial value. The number of minimums in Fig. $1 \mathrm{~b}$ depends on the recovery mechanisms governing the creep - polygonization for materials with high stacking fault energy (SFE) such as aluminum and recrystallization for low SFE (copper) [4]-[7].

a)

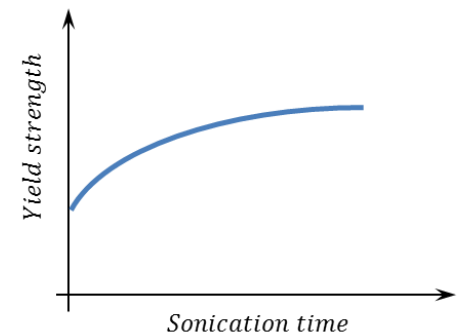

b)

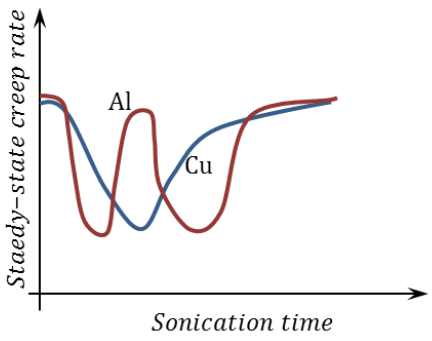

Figure 1

Schematic plots for the ultrasonic effects upon metals' properties-a) ultrasonic hardening, b) dependences of the creep rate upon the duration of preliminary sonication

(iii) Ultrasonic temporary softening manifests itself when a unidirectional loading is coupled with an oscillating one. Fig. 2 schematically demonstrates the effect of acoustic energy upon the development of plastic deformation in uniaxial tension. When the ultrasound is applied, the value of tensile stress decreases in a step-wise manner, and the further development of plastic deformation takes place at lesser value of the unidirectional stress. The stress-drop amount is measured to be proportional to the energy of acoustic energy or oscillating stress amplitude. Although the mechanism of the ultrasonic temporary softening is far from clear, researchers use the following suggestions or their combination:

a) A stress superimposition mechanism implies that ultrasonic waves activate anchored dislocations, hardened under ordinary deformation, and decrease stresses for further inelastic deformation,

b) An analogy between the effects of hot deformation and ultrasound action numerous researches suggest that ultrasonic vibration induces sufficient heat input to the sample to produce some softening of microstructure. [8]-[15]. 
(iv) Ultrasonic residual softening or hardening (Fig. 2) are observed after the ultrasound is Off, and the material deforms under the action of the static load alone. The appearance of one of the phenomenon depends on the changes in the material structure during acoustoplasticity. Thus, if acoustic energy induces dynamic recrystallization or other softening processes, the residual softening will be observed (Fig. 2a). In the case when, e.g., polygonized structure forms during the ultrasound-assisted deforming, more significant stress is needed for the further development of plastic deformation as ultrasound is Off (residual hardening, Fig. 2b) [16]-[18].

a)

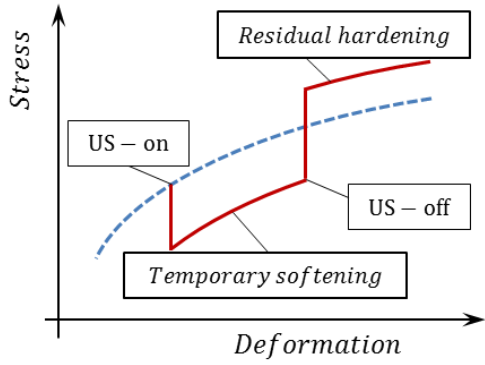

b)

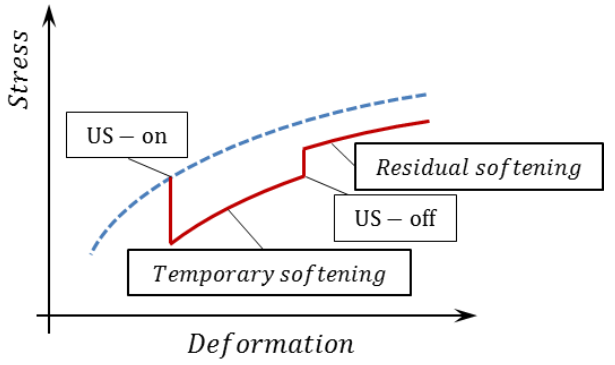

Figure 2

Schematic plots for the ultrasonic effects upon the plastic deformation: a) residual hardening,

b) residual softening

While the modeling of the phenomena above can be found in Rusinko's early works [1] [5] [19], the phenomenon discussed below so far was out of the authors' sight.

(v) Ultrasonic recovery effect. Acoustic energy has a recovery effect upon strain hardened materials.

Kulemin [4] conducted X-ray investigations to study the interference patterns evolution during the sonication of plastically deformed aluminum specimens ( $\varepsilon=5 \%$ ) at $20^{\circ} \mathrm{C}$ (Fig. 3). Due to the plastic deformation, clear interference spots of $0.3-1.0 \mathrm{~mm}$ radius of the annealed specimen blur out to $8 \mathrm{~mm}$ and 2-3 $\mathrm{mm}$ in the azimuthal and radial direction. After the ultrasonic action, the interference spot blurring decreases in both radial and polar directions, which testify to the relaxation of stresses of the second kind arisen due to the elastic distortions of crystalline grids in plastic deforming. Fig. 4 shows the decrease in the dislocation density, caused by the sonication at $20^{\circ} \mathrm{C}$ of the plastically hardened aluminum. As one can see, the dislocation density for the deformed material monotonically decreases to its initial value (annealed state) as a function of the sonication duration. 


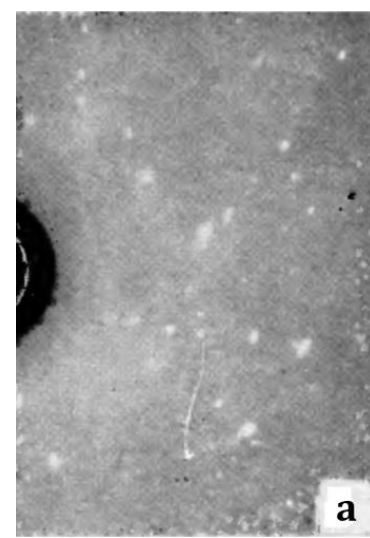

a

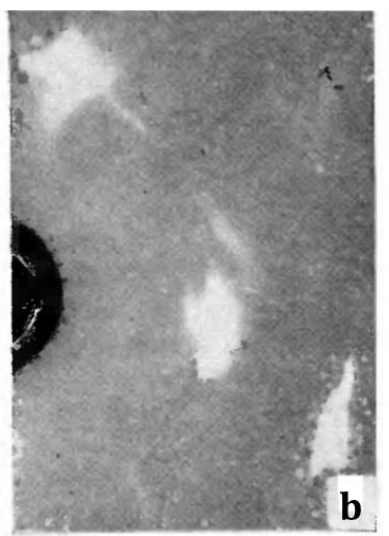

Figure 3

X-ray micrograph of aluminum specimen - a) annealed state, b) plastic deformation of 5\%, c) sonication of the deformed specimen; oscillating stress amplitude $16 \mathrm{MPa}$, duration $100 \mathrm{~min}$ [4]

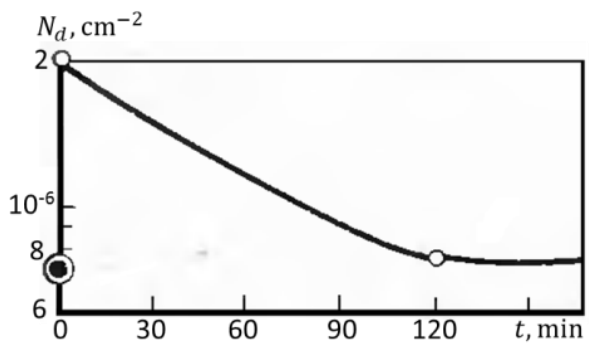

Figure 4

Dislocation density vs. sonication time at stress amplitude of $16 \mathrm{MPa}$ [4]

The ultrasonic recovery is suggested to proceed through polygonization when dislocations are built into low-angle boundaries. As a result, the initially distorted lattice is rearranging into stress-free blocks separated by the boundaries consisting of one sign's dislocations. The formation of the polygonized substructure requires the dislocations to leave their slip planes and climb parallel ones. As it is well known, the dislocation climb needs vacancies inflow which can be thermally activated as it is typical, for example, in elevated-temperature creep or during annealing. But since the results from Figs. 3 and 4 are obtained at room temperatures, the energy needed for the nucleation and migration of vacancies is provided by ultrasound. This result is harmonized with the established fact, that one of the features of sonication is an abundant number of point defects (vacancies). Fig. 5 confirms the above-considered thoughts about the dislocationclimb-nature of the ultrasonic recovering. Indeed, the straight slip lines from the plastic deformation (Fig. 5a) split into numerous intersected curved lines (Fig. 5b). 

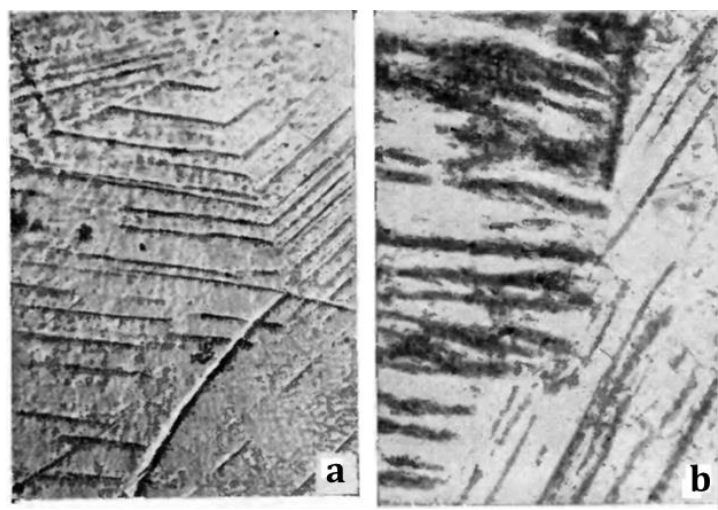

Figure 5

Slip lines on the surface of aluminum specimen - a) after plastic deformation, b) after the sonication of the plastically deformed specimen, $t=20^{\circ} \mathrm{C} . \times 400[4]$

Similar results were obtained at the sonication of cold-rolled steel strips [20], which indicate the yield strength decrease in the acoustic field.

With the intensity of the ultrasonic recovery, the following can be summarizing [4] [19]:

(i) There is a lower limit for the oscillating stress $\left(\sigma_{m}\right)$ beneath which the recovery effect is not observed (for example, while $\sigma_{m}=4.3 \mathrm{MPa}$ and $\sigma_{m}=5.6 \mathrm{MPa}$ gives no changes in the hardening-decrease, $\sigma_{m}=8.4 \mathrm{MPa}$ already yields the recovery effect [4])

(ii) The increase in $\sigma_{m}$ leads to a much steeper decrease of hardness/yield strength of the strain hardened material

(iii) At a given value $\sigma_{m}$, the acoustic energy causes more intensive recovery for more significant plastic deformations - the ultrasound-assisted recovery mechanisms are accelerating at the greater deformation energy cumulated in the material

(iv) with the sonications time, at significant values of $\sigma_{m}$, the ultrasonic recovery can be succeeded by some hardening (in a wave manner), but the overall trend remains unchangeable, decreasing hardness/yield strength. Such cases are not considered here.

Our paper is aimed to model the ultrasonic recovery effect in terms of the synthetic theory of irrecoverable deformation, which has already shown satisfactory results in the analytical description of ultrasonic softening and hardening [1] [5] [19] [21]. 


\section{The Synthetic Theory and Its Extensions for the Case of the Ultrasonic Recovery}

\subsection{Basic Equations}

In terms of this theory [20], plastic deformation at a point of the body - plastic strain deviator vector $(\overrightarrow{\mathbf{e}})$ - is determined via deformations at the micro-level of material, i.e., as a sum of plastic shifts in active slips systems where the Schmidt law conditions are met - the resolved shear stress exceeds the material yield strength:

$\overrightarrow{\mathbf{e}}=\iiint_{V} \varphi_{N} \overrightarrow{\mathbf{N}} d V$

$\varphi_{N}$ - plastic strain intensity - is an average measure of plastic deformation within one slip system.

The synthetic theory formulates a yield criterion and strain hardening rule in terms of three-dimensional stress deviator space $S^{3}$. It does not deal with a yield surface itself, but with its tangent planes, i.e., the yield surface is considered an inner envelope of the tangent planes. The location of planes is defined by their distances $\left(H_{N}\right)$ and unit normal vectors $(\overrightarrow{\mathbf{N}})$. For a virgin state, the synthetic theory works with the Von-Mises yield criterion, which results in the set of equidistant planes in all directions (Fig. 6a).

A stress vector $(\overrightarrow{\mathbf{S}})$ represents loading, whose coordinates are composed of the stress deviator tensor components. During the loading, the stress vector shifts at its endpoint a set of planes from their initial positions (Fig. 6b). The planes' movements at the endpoint of the stress vector are translational, i.e., the plane orientations keep changeless. Planes, which are not located at the endpoint of the vector $\overrightarrow{\mathbf{S}}$, remain stationary. The plane's displacement at the stress vector's endpoint represents a plastic flow within the corresponding slip system. 


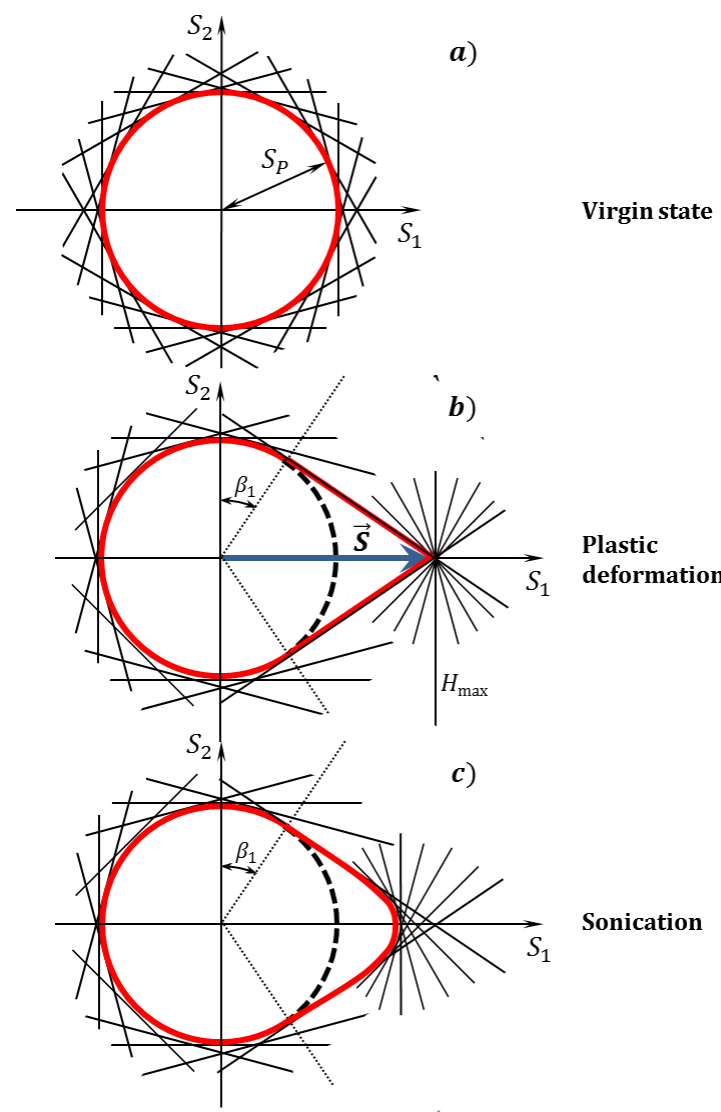

Figure 6

Yield and loading surface in terms of the synthetic theory in $S_{1}-S_{2}$ coordinate plane for uniaxial tension $(\lambda=0)$

We define the plastic strain intensity - the plastic flow rule on the micro-level of material - through the carriers of irrecoverable deformation, defect-intensity $\left(\psi_{N}\right)$ :

$d \psi_{N}=r d \varphi_{N}-K \psi_{N} d t$

where $r$ is the model constant determining the slope of the stress-strain diagram in its plastic portion, and $K$ is a function of temperature and acting stress, which governs the steady-state creep rate [20]:

$K=K_{1}(T) K_{2}\left(\tau_{0}\right)$, 


$$
\begin{aligned}
& K_{1}(T)=\exp \left(-\frac{Q}{R T}\right), \\
& K_{1}\left(\tau_{0}\right)=\frac{9 \sqrt{3} c r}{2 \sqrt{2} \pi} \tau_{0}^{k-2} .
\end{aligned}
$$

In the formulae above $Q$ is the thermal activation energy, $T$ is the temperature, $\tau_{0}$ is the Von-Mises stress, $c$ and $k$ are the model constants.

The hardening rule of a material is defined through the distances to the tangent planes

$$
\psi_{N}=H_{N}^{2}-S_{S}^{2}=\left\{\begin{array}{l}
(\overrightarrow{\mathbf{S}} \cdot \overrightarrow{\mathbf{N}})^{2}-S_{S}^{2} \text { for planes reached by } \overrightarrow{\mathbf{S}}: H_{N}=\overrightarrow{\mathbf{S}} \cdot \overrightarrow{\mathbf{N}} \\
0 \quad \text { for planes not reached by } \overrightarrow{\mathbf{S}}: H_{N}>\overrightarrow{\mathbf{S}} \cdot \overrightarrow{\mathbf{N}}
\end{array}\right.
$$

$S_{S}$ is a radius of the Von-Mises sphere; $S_{S}=\sqrt{2 / 3} \sigma_{S}$, where $\sigma_{S}$ is the yield strength of a material in uniaxial tension. It is clear from (6) that the greater plane distance is, the more significant stresses are needed to continue plastic deforming.

In uniaxial tension, the stress vector $\overrightarrow{\mathbf{S}}(\sqrt{2 / 3} \sigma, 0,0)$ extends along the $S_{1}$ axis, and the plane distances are

$$
H_{N}=S_{1} N_{1}=\sqrt{2 / 3} \sigma \sin \beta \cos \lambda
$$

For plastic deformation, when the time-dependent term in (2) can be neglected, we get from (2) and (6) the following expression

$$
r \varphi_{N}=\psi_{N}=(2 / 3)\left[(\sigma \sin \beta \cos \lambda)^{2}-\sigma_{S}^{2}\right]
$$

The loading surface consists of two parts: a) sphere as the envelope of stationary planes and b) cone whose generator is constituted by the boundary planes (angle $\beta_{1}$ in Fig. 6b) shifted by $\dot{\mathbf{S}}$.

Now, the integration in (1) gives the component of plastic strain vector component as

$e=\frac{4 \pi}{3 r} \int_{\beta_{1}}^{\pi / 2} \int_{0}^{\lambda_{1}}\left[(\sigma \sin \beta \cos \lambda)^{2}-\sigma_{S}^{2}\right] \sin \beta \cos \lambda \cos \beta d \lambda d \beta=a_{0} \Phi(b)$

where

$$
a_{0}=\frac{\pi \sigma_{S}^{2}}{9 r},
$$




$$
\begin{aligned}
& \Phi(b)=\frac{1}{b^{2}}\left[2 \sqrt{1-b^{2}}-5 b^{2} \sqrt{1-b^{2}}+3 b^{4} \ln \frac{1+\sqrt{1-b^{2}}}{b}\right], \\
& b \equiv \sin \beta_{1}=\frac{\sigma_{S}}{\sigma} .
\end{aligned}
$$

\subsection{Post-deformed State, Ultrasonic Recovery}

After the plastic strain is over $\left(d \varphi_{N}=0\right)$, Eq. (2) takes the following form

$$
d \psi_{N}=-K \psi_{N} d t
$$

The solution of the differential equation above gives

$$
\psi_{N}=\psi_{N 0} \exp (-K t)
$$

where $\psi_{N 0}$ reflects the number of defects cumulated in the material during plastic deforming, Eq. (8).

Formula (14), together with (6), means that the tangent planes start moving towards the origin of coordinates (Fig. 6c):

$$
H_{N}=\sqrt{\sigma_{S}^{2}+\frac{3}{2} \psi_{N 0} \exp (-K t)} .
$$

As it follows from (6) and (15), only the planes translated by the stress-vector during plastic deforming take part in the movement.

To adopt Eq. (14) for modeling the phenomenon that the acoustic energy leads to the recovery of work-hardened materials' mechanical properties, we propose the following.

Since the ultrasonic recovery experiments were held at the stress-free state, the function $K$ defined via formulae (3)-(5) is inapplicable because $K=0$ at $\tau_{0}=0$ and we obtain no change in $H_{N}$. Further, since the thermally activated processes at room temperature exert a feeble effect, we need to insert into the function $K$ a term that expresses the recovery processes induced by ultrasound.

We replace the function $K$ from (15) by

$$
K_{U}=K+A_{1}\left(\sigma_{m} H_{\max }\right)^{A_{2}},
$$

where $\sigma_{m}$ is the oscillating stress amplitude, $H_{\max }$ is the maximum plane distance for the whole loading history, $A_{1}$ and $A_{2}$ are the model constant to be chosen for the best fit between the analytic and experimental results. 
Therefore, now the degree of hardening - the plane distances in terms of the synthetic theory - obeys the following relationships:

$H_{N U}=\sqrt{\sigma_{S}^{2}+\frac{3}{2} \psi_{N 0} \exp \left(-A_{1}\left(\sigma_{m} H_{\max }\right)^{A_{2}} t\right)}$.

Let us analyze the modification proposed in Eqs. (16) and (17). The appearance of a term depending on the intensity of sonication $\left(\sigma_{m}\right)$ in the function that governs the decrease of the plane distances reflects the experimental fact that the acoustic energy can induce alone the recovery processes in plastically deformed materials. It is clear that $K_{U}=K$ in the absence of ultrasonic energy $\left(\sigma_{m}=0\right)$, i.e., we return to the case as with formula (3). Further, we defined $K_{U}$ via the product $\sigma_{m} H_{\max }$ to reflect another experimental fact that the greater values of strain hardening (plastic deformation) lead to more intensive recovery during the sonication at a given intensity of ultrasound and its duration. $H_{\max }$ is defined via formula (7) at $\beta=0$ and $\lambda=0$ :

$$
H_{\max }=S_{1} N_{1}=\sqrt{2 / 3} \sigma
$$

So $H_{\max }$, via $\sigma$, bears in itself the information on the degree of plastic deformation.

\subsection{Results and Discussion}

To inspect the modifications we proposed above, let us compare model results with experimental data [4] on the temporary decrease of Vickers hardness number $(H V)$ of aluminum specimen plastically deformed in uniaxial tension on two values: $\varepsilon_{1}=3.6 \%$ and $\varepsilon_{2}=6.8 \%$ (Fig. 7). The sonication of the strain hardened specimen is conducted by the longitudinal oscillation of amplitude $\sigma_{m}=10 \mathrm{MPa}$.

Both the plastic deforming and the sonication take place at room temperature. To relate the value of $H V$ to the yield strength in uniaxial tension $\sigma_{S}$, we address the results on the correlation between Vickers hardness number and yield strength for aluminum, which states that $H V=17.4$ corresponds to $\sigma_{S}=23.4 \mathrm{MPa}$ [22]. We preserve the relation $R \equiv H V / \sigma_{S}=0.744$ not only for the start of plastic deforming but for formula (17) as well:

$$
H V=R \cdot \sqrt{\sigma_{S}^{2}+\frac{3}{2} \psi_{N 0} \exp \left(-A_{1}\left(\sigma_{m} H_{\max }\right)^{A_{2}} t\right)} .
$$

To apply the formula above, first, we need to choose the constant $r$ to make sure that it gives correct $H V$ for the plastically deformed specimens. For this purpose,

(i) We utilize formulae (9)-(12), which give $\varepsilon \sim \sigma$ relation 
(ii) From the $\varepsilon \sim \sigma$ relationships, we take those values of the stresses ( $\sigma_{1}=31.0 \mathrm{MPa}$ and $\sigma_{2}=33.6 \mathrm{MPa}$ ) that correspond to $\varepsilon_{1}=3.6 \%$ and $\varepsilon_{2}=6.8 \%$

(iii) On the base of (8), we calculate $\psi_{N 0}$ for $\sigma_{1}$ and $\sigma_{2}$

(iv) Formula (19) at $t=0$ gives the values of $H V$ for the plastic deformation caused by $\sigma_{1}$ and $\sigma_{2}$

As a result, we obtain two points on the $H V \sim t$ diagram at $t=0$

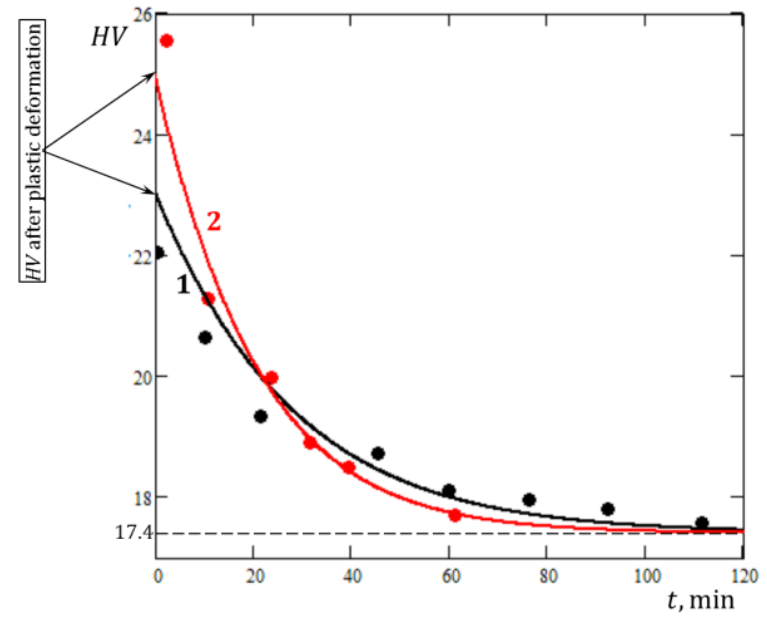

Figure 7

$H V$ vs. sonication time plots for the plastically deformed aluminum specimen: $\varepsilon_{1}=3.6 \%$ and

$$
\varepsilon_{2}=6.8 \% \text {. - experiment [4], lines - model }
$$

The next step is to model the decrease in $H V$ as a function of sonication time by formula (19). Fig. 7 demonstrates the model $H V \sim t$ curves constructed at the following constants' values: $A_{1}=7.136 \times 10^{14}\left(\mathrm{MPa}^{2 A_{2}} \cdot \mathrm{s}\right)^{-1}, A_{2}=4.0$. As in the experiment, the model curve at $\varepsilon_{2}=6.8 \%$ shows a quicker decrease in $H V$ than that at $\varepsilon_{1}=3.6 \%$. It is in full accordance with the experimental fact that the initial strain hardening increase boosts the acoustic field's recovery processes.

\section{Conclusions}

In this work we developed a model that analytically describes the phenomenon of ultrasonic recovery. For the mathematical apparatus, we utilized the synthetic theory of irrecoverable deformation, which has shown itself to be an effective tool for modelling the various problems that are related to ultrasound-assisted processes. We extend the synthetic theory, via the introduction of a new term 
accounting, for the presence of oscillating stress. As a result, we obtained a plot for the micro-hardness of plastically deformed aluminum specimens, that are subjected to sonication. The results show a good agreement with the experimental data.

\section{Acknowledgment}

This work was supported by Doctoral School on Materials Science and Technologies, ID 215, Óbuda University.

\section{References}

[1] Rusinko, A.: Analytical description of ultrasonic hardening and softening. Ultrasonics, 51, 709-714, 2011

[2] Peslo, A.: Ultrasonic hardening of aluminium alloys. Ultrasonics, 22, 37 41, 1984

[3] A. Kozlov, N. Mordyuk: The influence of preliminary ultrasound treatment on the strength of metals, Ultrasonic vibrations and their impact upon the mechanical characteristics of metals, Kiev, 1986, pp. 31-34

[4] Kulemin, A.: Ultrasound and Diffusion in Metals. Moscow, Metallurgia, 1978

[5] Rusinko, A.: Influence of preliminary ultrasonic treatment upon the steadystate creep of metals of different stacking fault energies. Ultrasonics, 54, 90-98, 2014

[6] Bazeljuk, G. et al.: Influence of preliminary ultrasonic irradiation and mechanics-thermal treatment upon the high-temperature creep and microhardness of copper, Fizika metallov i metallovedenije, 29: 508-511, 1970

[7] Bazeljuk, G. et al.: Influence of preliminary ultrasonic irradiation and mechanics-thermal treatment upon the creep-resistance of aluminum, Fizika metallov i metallovedenije, 32: 145-151, 1971

[8] Bagherzadeh, S. \& Abrinia, K.: Effect of ultrasonic vibration on compression behavior and microstructural characteristics of commercially pure aluminum. Journal of Materials Engineering and Performance, 24, 4364-4376, 2015

[9] Blagoveshchenskii, V., Panin, I.: An increase in the rate of plastic deformation under the effect of ultrasound. The Physics of Metals and Metallography, 103, 424-426, 2007

[10] Daud, Y., Lucas, M. \& Huang, Z.: Modelling the effects of superimposed ultrasonic vibrations on tension and compression tests of aluminium. $J$. Mater. Process. Technol, 186, 179-190, 2007 
[11] Deshpande, A., Tofangchi, A., \& Hsu, K.: Microstructure evolution of Al6061 and copper during ultrasonic energy-assisted compression. Materials Characterization, 153, 240-250, 2019

[12] Geibler, U., Schneider-Ramelow, M., \& Reichl, H.: Hardening and softening in AlSi1 bond contacts during ultrasonic wire bonding. IEEE Transactions on Components and Packaging Technologies, 32, 794-798, 2009

[13] Graff, K.: Ultrasonic metal forming: processing. Power Ultrasonics, Edited by Juan A. Gallego and Karl F. Graff, Woodhead Publishing, pp. 377-438, 2015

[14] Huang, H., Pequegnat, A., Chang, B., Mayer, M., Du, D. \& Zhou, Y.: Influence of superimposed ultrasound on deformability of $\mathrm{Cu}$. Journal of Applied Physics, 106, 113514, 2009

[15] Malygin, G. A.: Acoustoplastic effect and the stress superimposition mechanism. Physics of the Solid State, 42, 72-78, 2000

[16] Yao, Z., Kim, G. Y., Wang, Z., Faidley, L., Zou, Q., Mei, D., \& Chen, Z.: Acoustic softening and residual hardening in aluminum: modeling and experiments. International Journal of Plasticity, 38, 75-87, 2012

[17] Zhou, H., Cui, H., and Qin, Q.: Influence of ultrasonic vibration on the plasticity of metals during compression process. J. Mater. Process. Technol., 251, 146-159, 2018

[18] Lum, I., Huang, H., Chang, B., Mayer, M., Du, D., and Zhou, Y.: Effects of superimposed ultrasound on deformation of gold. Journal of Applied Physics, 105, 024902, 2009

[19] A. Rusinko, Ali H. Alhilfi: Evolution of loading surface in the ultrasonic field, Proceedings of the Engineering Symposium at Bánki, Budapest 2020, pp. $35-40$

[20] Susan, M. M., Dumitras, P. G. and Iliescu, V. G.. Experimental research of the ultrasonic softening in cold-rolled steel strips. Электронная обработка материалов №1, 2007

[21] Rusinko, A. \& Rusinko, K.: Plasticity and Creep of Metals. Berlin, Springer, 2011

[22] Arbtin Jr., E., Murphym G.: Correlation of Vickers hardness number, modulus of elasticity, and the yield strength for ductile metals. Ames Laboratory ISC Technical Reports, 1953 